2017-01

The Neural Correlates of Similarity- and Rule-based Generalization

Milton, F

http://hdl.handle.net/10026.1/5435

10.1162/jocn_a_01024

Journal of Cognitive Neuroscience

MIT Press - Journals

All content in PEARL is protected by copyright law. Author manuscripts are made available in accordance with publisher policies. Please cite only the published version using the details provided on the item record or document. In the absence of an open licence (e.g. Creative Commons), permissions for further reuse of content should be sought from the publisher or author. 


\title{
The Neural Correlates of Similarity- and Rule-based Generalization
}

\author{
Fraser Milton ${ }^{1}$, Pippa Bealing ${ }^{1}$, Kathryn L. Carpenter ${ }^{1}$, \\ Abdelmalek Bennattayallah ${ }^{1}$, and Andy J. Wills ${ }^{2}$
}

\begin{abstract}
The idea that there are multiple learning systems has become increasingly influential in recent years with many studies providing evidence that there is both a quick, similarity-based, or feature-based system and a more effortful, rule-based system. A smaller number of imaging studies have also examined whether neurally dissociable learning systems are detectable. We further investigate this by employing for the first time in an imaging study a combined positive and negative patterning procedure originally developed by Shanks and Darby [Shanks, D. R., \& Darby, R. J. Feature- and rule-based generalization in human associative learning. Journal of Experimental Psychology: Animal Behavior Processes, 24, 405-415, 1998]. Unlike previous related studies employing other procedures, rule generalization
\end{abstract}

\section{INTRODUCTION}

The ability to generalize information we have previously learned to novel stimuli is fundamental for successful functioning in our everyday environment. An enduring and contentious question is whether this is achieved by separable learning systems (e.g., Ashby, Alfonse-Reese, Turken, \& Waldron, 1998; Brooks, 1978) or just a single system (e.g., Newell, Dunn, \& Kalish, 2011; Nosofsky \& Kruschke, 2002). Multiple-system accounts typically posit the existence of a nondeliberative (Wills, Milton, Longmore, Hester, \& Robinson, 2013) or nonanalytic (Brooks, 1978) process, that is automatic (Smith, Patalano, \& Jonides, 1998), similarity-based (Milton, Longmore, \& Wills, 2008), and driven by associative (McLaren, Green, \& Mackintosh, 1994) or implicit (Ashby et al., 1998) processes. A second system is assumed to be deliberative (Wills et al., 2013) or analytic (Brooks, 1978), controlled (Smith et al., 1998), rulebased (Ashby et al., 1998), and requiring of extensive cognitive resources (Wills, Inkster, \& Milton, 2015). In this article, we refer to these two systems as similarity- and rule-based.

Much of the evidence relevant to this debate has come from behavioral or comparative studies. Some of this evidence is consistent with multiple learning systems accounts (e.g., Maes et al., 2015; Ashby \& Maddox, 2011; Allen \&

\footnotetext{
${ }^{1}$ University of Exeter, ${ }^{2}$ University of Plymouth
}

in the Shanks-Darby task is beyond any simple non-rule-based (e.g., associative) account. We found that rule- and similaritybased generalization evoked common activation in diverse regions including the $\mathrm{pFC}$ and the bilateral parietal and occipital lobes indicating that both strategies likely share a range of common processes. No differences between strategies were identified in whole-brain comparisons, but exploratory analyses indicated that rule-based generalization led to greater activation in the right middle frontal cortex than similarity-based generalization. Conversely, the similarity group activated the anterior medial frontal lobe and right inferior parietal lobes more than the rule group did. The implications of these results are discussed.

Brooks, 1991; Rips, 1989; Kemler Nelson, 1984), whereas others maintain that this evidence can be more parsimoniously explained by a single system (e.g., Edmunds, Milton, \& Wills, 2015; Wills et al., 2015; Newell, Moore, Wills, \& Milton, 2013; Stanton \& Nosofsky, 2013). Consequently, there is currently no clear consensus on this issue. A complimentary and currently relatively underexplored approach is to use brain imaging to examine whether there are neurally dissociable learning systems. One such fMRI study, loosely based on earlier behavioral work by Allen and Brooks (1991), was conducted by Koenig et al. (2005), who asked participants to classify a set of cartoon animals differing on four stimulus dimensions (e.g., legs, neck type). Participants in the rule condition were informed of a complex rule (category membership requires the instance to possess three of four characteristic features for that category). In the similarity condition, participants were not told the rule but instead asked to make a quick decision using their first impressions about which category a particular instance was more similar to. Both groups were provided with trial-by-trial feedback. Koenig et al. found that similarity-based, compared with rule-based, categorization recruited greater activation in bilateral temporoparietal regions as well as bilateral anterior prefrontal regions (BA 10). Conversely, the rule-based condition led to greater activation than the similarity condition in the left frontal lobes, left inferior parietal 
lobes, and the right superior parietal lobes. One feature of this study, however, is that it is not clear exactly what strategy participants in the similarity condition are employing, which makes interpretation of the imaging results more complicated. Specifically, Koenig et al. assume that participants are using a similarity-based approach that presumably requires the use of most, if not all, of the dimensions. Although this is plausible, an alternative explanation is that participants in the similarity condition are using a simpler rule-based approach, such as a single dimension-plus-exception strategy (e.g., Ward \& Scott, 1987), which could also result in the level of performance obtained. In this latter case, participants in the similarity condition are using fewer of the dimensions than those in the rule condition.

In a study somewhat more closely based on the work of Allen and Brooks (1991), Patalano, Smith, Jonides, and Koeppe (2001) also observed activation in bilateral frontal cortex in the rule-based condition that was not present in the similarity condition. Occipital lobe and cerebellum activation was prevalent in both conditions. However, significant neural differences between the groups were relatively restricted, even though one-tailed tests were used. For example, the greater frontal lobe activation in the rule than the similarity condition was only marginally significant $(p=.06)$.

Using a slightly different approach - the criterial attribute procedure, based on earlier behavioral work by Kemler Nelson (1984) — Tracy et al. (2003) investigated the neural correlates of family resemblance categorization (assumed to use the similarity system) and unidimensional categorization (assumed to employ the rule system). Similar to the category structure employed by Koenig et al. (2005), a family resemblance category (e.g., Rosch \& Mervis, 1975) possessed a number of characteristic but not defining features - an item did not have to possess any single feature or features as long as it possessed enough characteristic features (three of four typical features) of that category. In contrast, a unidimensional category was based around a single defining feature that the authors assumed required use of the rule-based system. Tracy et al. (2003) found greater activation in the extrastriate cortex (BA 18 and BA 19) and the left cerebellum for family resemblance (similarity-based) categorization than unidimensional categorization, whereas unidimensional categorization led to greater activation in bilateral frontal lobes than family resemblance categorization. However, recent behavioral model-based analysis suggests that family resemblance categorization in the criterial attribute procedure is often due to the use of a single noncriterial dimension, which is a strategy not detectable by the standard analysis employed by Tracy et al. (for a detailed discussion, see Wills et al., 2015). This again makes interpretation of the neural differences observed more difficult.

In contrast to Tracy et al.'s (2003) conclusions, Milton, Wills, and Hodgson (2009) proposed that both family resemblance and unidimensional categorization are the result of a single rule-based system, with family resemblance categorization requiring a more complex, multidimensional rule than unidimensional categorization (see also Wills et al., 2013). Consistent with their proposal, Milton et al. found extensive common activation between family resemblance and unidimensional categorization including the dorsolateral frontal cortex and the anterior cingulate. The most notable difference between groups was the greater right ventrolateral frontal cortex activation for family resemblance than unidimensional categorization, which the authors proposed indicated the greater working memory resources required to employ a multidimensional rule.

A different approach was taken by Nomura et al. (2007), who conducted an fMRI study based on the influential COVIS framework (Ashby et al., 1998); participants viewed a series of Gabor patches and learned either a rule-based task that possessed an easily verbalizable, unidimensional rule ("thinner lines belong in category A, thicker lines in category B"), which is assumed to encourage use of the explicit system or an informationintegration task, which requires participants to combine information from two unrelated stimulus dimensions. The optimal information-integration category structure is assumed to be difficult or impossible to verbalize, which should encourage use of COVIS's implicit system. In line with COVIS's predictions, dissociable neural activation was found with the medial-temporal lobes more activated in rule-based compared with information-integration learning, and the caudate body more engaged in information-integration than rule-based learning.

Although intriguing, the category separation (i.e., the mean distance between category items as plotted in stimulus space divided by the within-category variance along the direction of the comparison) was smaller in the rulebased than the information-integration condition, and the selective attention demands were greater in the rule-based than the information-integration condition (as only one of the two dimensions was relevant to learn the rule-based structure whereas both were required for the information-integration structure) meaning that nonessential differences could have been driving the neural dissociations. In a recent study conducted by Carpenter, Wills, Benattayallah, and Milton (in press) when these nonessential differences between the rule-based and information-integration conditions were better equated (by comparing a conjunctive rule-based structure against a standard information-integration structure), the pattern of results observed by Nomura et al. (2007) did not emerge, and instead there was extensive common overlap between the conditions. Furthermore, the information-integration condition evoked greater activation in the medial-temporal lobes than the rule-based condition, which may reflect the greater memory demands in the information-integration condition where no rule was readily available. In another related study, albeit one 
which used very different stimuli (the stimuli varied on rectangle height and width of an ellipse), Milton and Pothos (2011) compared activation between a rule-based task and an information-integration-like task but found minimal neural dissociations and instead found extensive overlap of activation suggesting that both groups were using similar neural processes.

Finally, Grossman et al. (2002), using a modified version of Rips's (1989) classic procedure, gave participants a description of an item such as "a round object 2 inches in diameter" who had to assign it to either the category of "quarter" or "pizza." The description is more similar in size to a quarter than a pizza but a pizza has a variable diameter (so could, in principle, be 2 inches) whereas a quarter does not (so it cannot be 2 inches). Participants who choose the quarter category were assumed to be making a similarity judgment, whereas those who assign it to the pizza category are following a rule. Grossman et al. found that there was greater recruitment of the left dorsolateral $\mathrm{pFC}$ for rule than similarity responses whereas the right inferior parietal lobe, which they noted is involved in overall feature configuration (Wilkinson, Halligan, Henson, \& Dolan, 2002), was activated more for similarity- than rule-based responses. However, Nosofsky and Johansen (2000) have demonstrated that the results from this procedure can be accommodated by a simple, single-process, exemplar-based learning system without requiring qualitatively distinct systems for the different strategies.

We investigate whether there are neurally separable rule and similarity generalization systems from a different angle using a procedure based on Shanks and Darby's (1998) Experiment 2, which has not previously been examined using brain imaging. The design of this experiment is shown in Figure 1. Participants took the role of an allergist who had to determine whether the meals a hypothetical patient, Mr. X, eats will cause an allergic reaction or not. Letters in Figure 1 stand for particular foods (e.g., pasta or eggs), + indicates that an allergic reaction will develop, and - indicates that no allergic reaction will occur. During training, participants learn two complete negative patterning problems (e.g., $\mathrm{A}+$,

\begin{tabular}{|llllll|}
\hline & Training & & & Test & \\
A+ & B+ & AB- & A? & B? & AB? \\
C- & D- & CD+ & C? & D? & CD? \\
E+ & F+ & EF- & E? & F? & EF? \\
G- & H- & GH+ & G? & H? & GH? \\
I+ & J+ & IL- & I? & T? & IJ? \\
& & KL & L? & KL? \\
M- & N- & MN & M? & N? & MN? \\
& & OP+ & O? & P? & OP? \\
\hline
\end{tabular}

Figure 1. The training and test trial types in the Shanks and Darby (1998, Experiment 2) allergy prediction task; letters indicate foods eaten by a hypothetical patient $\mathrm{Mr} . \mathrm{X},+=$ patient develops an allergic reaction; $-=$ patient does not develop an allergy reaction; ? = no feedback given
$\mathrm{B}+, \mathrm{AB}-)$ and two complete positive patterning problems (e.g., $\mathrm{C}-, \mathrm{D}-, \mathrm{CD}+$ ). Critically, however, there are also four incomplete patterning problems-for example, participants are trained on $\mathrm{I}+$ and $\mathrm{J}+$ but not on the outcome of I and $\mathrm{J}$ combined and trained that eating $\mathrm{KL}$ together leads to an allergic reaction but not what happens when $\mathrm{K}$ and $\mathrm{L}$ are eaten separately. During the test phase, as well as being tested on items they studied during training (e.g., $\mathrm{I}+, \mathrm{J}+$, and $\mathrm{KL}+$ ), participants have to generalize the knowledge they have obtained to what will henceforth be referred to as the critical items (e.g., IJ, K, and L; shown in bold in Figure 1) and are provided no feedback on their responses.

In the case of IJ, if participants are using a similaritybased strategy then they should predict an allergic reaction as it is similar to I and J, both of which lead to an allergic reaction alone. Equally, when presented with $\mathrm{K}$ or $\mathrm{L}$ alone they should predict an allergic reaction because they are similar to KL which results in an allergic reaction. In contrast, if participants have learned the "opposites" rule from training-single foods predict the opposite to their compounds-they can use this to generalize to novel items. In this case, IJ should lead to no allergic reaction, because it is the opposite outcome to I or J when presented alone. Similarly, K or L, when presented separately, should lead to no allergic reaction as this is the opposite to $\mathrm{KL}$, which resulted in an allergic reaction. Shanks and Darby (1998) found that participants with high accuracy during training produced more rule-based responses for these critical test items than participants with lower accuracy. They explained this by postulating that there is a transition from a similarity to a rule-based approach, which can only be used when the basic associations have been acquired (see also Wills, Graham, Koh, Mclaren, \& Rolland, 2011).

While using a novel procedure to compare the neural correlates of the purported rule and similarity systems is of value in itself, the Shanks-Darby procedure has some particular advantages that make it well equipped to provide new insight into this debate. First, both similarityand rule-based responses require utilizing the same number of stimulus dimensions. This is in contrast to many of the studies described above (e.g., Milton \& Pothos, 2011; Milton et al., 2009; Nomura et al., 2007; Tracy et al., 2003) where the number of stimulus dimensions utilized in the similarity condition seem unlikely to be the same as in the rule conditions (it may either be more, as is commonly assumed, or sometimes less, depending on how participants approach the task in the similarity condition). Across a range of different procedures, categorizing by a larger number of dimensions is more effortful than categorizing by fewer dimensions (e.g., Edmunds et al., 2015; Wills et al., 2015; Milton \& Wills, 2004). It is plausible that this could be driving the difference in neural activation between the groups, rather than indicating the involvement of qualitatively different systems. A further advantage of employing the Shanks-Darby procedure is 
that a full explanation of the "opposites" rule generalization is commonly thought to be beyond published associative accounts (see Maes et al., 2015, for a further discussion) allowing clear inferences to be drawn. This does not appear to be the case for the other procedures described above. For instance, non-rule-based accounts, such as Kruschke's (1992) ALCOVE model, which have a mechanism for dimensional attention, are able to account for the purported rule-based classification in these tasks.

In summary, then, this study uses a novel approach to investigate the neural differences between rule-based and similarity-based generalization. We predicted, based on previous behavioral and comparative work with this procedure (Maes et al., 2015; Wills et al., 2011), that we would observe neural differences between the generalization strategies. In particular, we hypothesized that there would be greater frontal lobe activation in the rule-based condition than the similarity-based condition (e.g., Milton et al., 2009; Nomura \& Reber, 2008; Grossman et al., 2002; Patalano et al., 2001). Our prediction for which regions would be implicated in similarity-based generalization was more tentative given the greater heterogeneity in previous studies but viable options a priori included the right inferior parietal lobes (Grossman et al., 2002) and the occipital lobes (e.g., Nomura et al., 2007; Patalano et al., 2001)/extrastriate cortex (Tracy et al., 2003).

\section{METHODS}

\section{Participants}

Sixty-two right-handed participants were recruited from the University of Exeter participant pool. Participants were either volunteers, received course credits, or were paid §7. Participants all gave informed consent according to procedures approved by the Psychology Ethics Committee, University of Exeter. A learning criterion was set as significantly above chance accuracy in the second half of training to ensure that all participants included in the analyses had clear evidence of learning. Without this, one could not reasonably expect true generalization to occur. This resulted in the exclusion of 10 participants. A further 14 participants did not show clear evidence of either rule- or similarity-based generalization-defined as significantly above chance (64.6\%) strategy-consistent responding for the critical test trials. These participants who did not adopt a clear strategy would likely obscure any differences that emerged between participants who did demonstrate clear rule or similarity generalization so we consequently excluded them from all the principal analyses. We, however, consider the test phase data for these 14 participants who used a mixture of rule and similarity consistent responses separately. This left 38 participants in total for our principal analyses; 24 rule-based responders and 14 similarity-based responders. This trend for a greater proportion of rule responders was not significant, $\chi^{2}(1)=2.632, p=.105$.

\section{Stimuli}

The stimuli (food names) were identical to those used in Experiment 2 of Shanks and Darby (1998). For half of the participants, the food names A-P (see Figure 1) were cheese, garlic, milk, mushrooms, seafood, red meat, olive oil, coffee, banana, eggs, orange squash, bread, avocado, peanuts, pasta, and chocolate. For the other half, the foods assigned to $\mathrm{A} / \mathrm{B}$ were exchanged for those assigned to $\mathrm{C} / \mathrm{D}$ and likewise for $\mathrm{E} / \mathrm{F}$ and $\mathrm{G} / \mathrm{H}, \mathrm{I} / \mathrm{J}$ and $\mathrm{K} / \mathrm{L}$, and $\mathrm{M} / \mathrm{N}$ and $\mathrm{O} / \mathrm{P}$.

\section{Procedure}

Before entering the scanner, participants were asked to take the role of a food allergist and to learn when Mr. X would develop an allergic reaction after eating a meal containing certain foods. Twenty-nine participants were additionally provided with instructions outlining the rule (e.g., "If Mr. X is allergic to a food when it is presented on its own, he won't be allergic to it when it is presented together with another food. Conversely, if $\mathrm{Mr}$. $\mathrm{X}$ is not allergic to a food when it is presented on its own then he will be if it is presented in combination with another food"), and 33 participants were provided with instructions designed to encourage a similarity-based approach ("When making a response, please use your intuition as to what you feel is the correct answer based on what you have previously seen"). The rationale behind this was to facilitate obtaining a sufficient number of participants who consistently categorized the critical trials by either a similarity or a rule-based approach rather than to look at the neural effects of differing instructions per se. In practice, however, this instructional manipulation had no significant impact on the strategy used (we suspect, in hindsight, that it would have been more effective if, like the training items, it had been presented inside the scanner in the same context as where learning took place) and will, consequently, not be discussed further.

Visual stimuli were presented on a back-projection screen positioned at the foot end of the MRI scanner and viewed via a mirror mounted on a head coil. Buttonpress responses and RTs were measured using a fiberoptic button box. E-Prime (Psychological Software Tools, 2002, www.pstnet.com) was used for the presentation and timing of stimuli and collection of response data.

In the training phase, participants received six blocks of trials, divided into two scanning runs of three blocks, with each of the 18 training stimuli (see Figure 1) presented twice in each block in a random order. Each trial began with a white screen lasting a random interval between 500 and $4000 \mathrm{msec}$, before a black fixation cross was presented in the middle of the screen for $250 \mathrm{msec}$. A meal, food names presented in black font, was then presented in the middle of a white screen for $3000 \mathrm{msec}$ during which time participants indicated whether it would lead to an allergic reaction (by pressing the left 
button box key) or would not lead to an allergic reaction (by pressing the right button box key). Following this, feedback ("Correct," in blue or "Incorrect," in red) was presented for $500 \mathrm{msec}$. For participants who failed to respond when the meal was presented, the message "Timeout!!" (in red) appeared instead. The next trial then immediately began. At the end of each block of 36 trials, the average accuracy on that block was displayed on the screen for $12 \mathrm{sec}$, before the next block began.

The test phase included all 24 stimuli, comprising the 18 training stimuli plus the six critical generalization stimuli (shown in bold in Figure 1). The 18 training stimuli were presented once in each block, whereas the six critical trials were each presented twice leading to 30 trials in each of the four blocks. Stimuli were presented in a random order. The intratrial structure was identical to the training phase, except that after a response a 500-msec blank screen appeared rather than feedback. If no response was made, a time out message appeared as in the training phase.

\section{fMRI Data Acquisition}

Images were collected using a 1.5-T Phillips Gyroscan magnet equipped with a Sense coil. A T2*-weighted echoplanar sequence was used (repetition time $=3000 \mathrm{msec}$, echo time $=45 \mathrm{msec}$, flip angle $=90^{\circ}, 32$ transverse slices, field of view $=240 \mathrm{~mm}, 3.5 \times 2.5 \times 2.5 \mathrm{~mm}$ ). The training phase comprised two runs of 240 scans, and the test phase one run of 260 scans. Five dummy scans were performed before the start of each stimulus sequence. Standard volumetric anatomical MRI was performed after functional scanning by using a 3-D T1-weighted pulse sequence (repetition time $=25 \mathrm{msec}$, echo time $=4.1 \mathrm{msec}$, flip angle $=30^{\circ}, 160$ axial slices, $1.6 \times 0.9 \times 0.9 \mathrm{~mm}$ ).

\section{Analysis of fMRI Data}

Analyses were carried out using SPM8 software (www.fil. ion.ucl.ac.uk/spm). Functional images were corrected for acquisition order, realigned to the mean image, and resliced to correct for motion artifacts. The realigned images were coregistered with the structural T1 volume, and the structural volumes were spatially normalized. The spatial transformation was applied to the realigned T2* volumes, which were spatially smoothed using a Gaussian kernel of $8 \mathrm{~mm}$ FWHM. Data were high-pass filtered $(1 / 128 \mathrm{~Hz})$ to account for low-frequency drifts. The BOLD response was modeled by a canonical hemodynamic response function.

All analyses were conducted using the general linear model. In the individual participant models, the critical trials that were consistent with their overall favored strategy (i.e., rule- or similarity-based generalization) were included as one regressor, whereas critical trials inconsistent with this approach were a second regressor. The familiar items were partitioned into correct and in- correct responses. The duration of each event was modeled as the participant's RT for that trial (see Grinband, Wager, Lindquist, Ferrera, \& Hirsch, 2008, for the advantages of using this "variable epoch" approach). Time outs were included as a fifth regressor of no interest. The six head movement parameters were included as additional covariates. Contrasts comparing strategy-consistent responses for the critical trials were subtracted against the implicit baseline (the intervals between the five event types listed above; cf. Milton et al., 2009; Tracy et al., 2003, for a similar approach), and correct familiar trials were likewise compared with the implicit baseline. These comparisons were then included in random-effects analyses. For these analyses, participants were divided into those who provided clear evidence of either similarity or rule-based generalization (i.e., significantly above chance strategy-consistent responding on the critical generalization trials).

Whole-brain analyses were completed using a combined statistical threshold of $p<.001$ (uncorrected) and a threshold of 100 contiguous voxels, which together produce an overall corrected threshold of $p<.05$. These values were estimated using $3 \mathrm{dClustSim}$ as implemented in the AFNI toolbox (afni.nimh.nih.gov/afni/). For this, we used a smoothness estimate of $10.1 \times 10.1 \times 9.6 \mathrm{~mm}$ (this was a group level estimate calculated in SPM8 using the group residuals from the general linear model, e.g., Kiebel, Poline, Friston, Homes, \& Worsley, 1999). In addition, to measure common activation between rulebased and similarity-based participants, conjunction analyses were performed. To do this, the relevant contrasts were combined using a logical "and" function through the minimum statistic to the conjunction null hypothesis (MS/CN; Nichols, Brett, Andersson, Wager, \& Poline, 2005) technique implemented in SPM8. Both contrasts were again conducted with a combined threshold of $p<.001$ (uncorrected) and a cluster threshold of 100 contiguous voxels. Note that this analysis is conservative because it reveals only those regions significantly activated for both the rule ( $p<.05$, corrected) and the similarity $(p<.05$, corrected) conditions.

After performing the whole-brain analyses, we decided to conduct more exploratory ROI analyses (using the WFU Pickatlas, e.g., Maldjian, Laurienti, Burdette, \& Kraft, 2003) when directly comparing rule and similarity generalization. These post hoc ROI analyses were based on our a priori predictions of regions we thought would be differentially involved between strategies and comprised the pFC (e.g., Milton \& Pothos, 2011; Milton et al., 2009), the occipital lobes/extrastriate cortex (BA 18 and BA 19; e.g., Nomura et al., 2007; Tracy et al., 2003), and the right inferior parietal lobes (Grossman et al., 2002). Although these exploratory analyses should accordingly be taken with some caution, we believe that they help to characterize better the nature of our results, which is particularly important given that this is the first imaging study of the Shanks-Darby procedure. For these analyses, we 


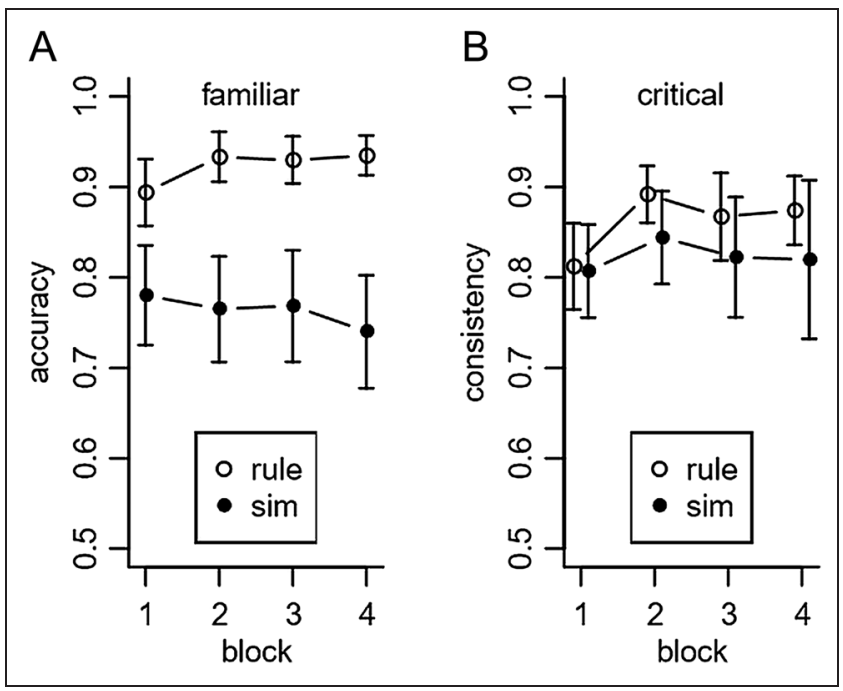

Figure 2. (A) Accuracy of rule-based and similarity-based responders for familiar items during the test phase. (B) Proportion of critical trials across blocks which are consistent with the strategy participants were assigned to.

used thresholds of $p<.001$ and 64 contiguous voxels, which together produce an overall corrected threshold of $p<.05$, as estimated by 3dClustSim. Normalized MNI space coordinates were transformed to Talairach space (imaging.mrccbu.cam.ac.uk/imaging/MniTalairach) to establish activation sites as per the atlas of Talairach and Tournoux (1988). ${ }^{1}$

\section{RESULTS}

\section{Behavioral Analyses}

Training Phase

The proportions of timeouts were low in both the rule $(M=.015, S D=.021)$ and similarity $(M=.020, S D=$ .018) groups, and there was no significant difference between them, $t(31.1)=.829, p=.413$. One-sample $t$ tests revealed that the average performance in the second half of training (Blocks 4-6) was significantly above chance for both the rule-based $(M=.872, S D=.092 ; t(23)=$ $19.647, p<.001)$ and the similarity-based $(M=.739$, $S D=.088 ; t(13)=10.217, p<.001)$ groups, although, as in Shanks and Darby (1998), the rule group had higher accuracy than the similarity group, $t(28.6)=4.409, p<$ .001 . Median RTs were longer in the similarity group $(1291 \mathrm{msec})$ than in the rule group $(1010 \mathrm{msec}), t(21.8)=$ $3.874, p<.001$.

\section{Test Phase}

The proportions of timeouts were again low (rule group: $M=.012, S D=.016$; similarity group: $M=.026, S D=$ $.031)$, and there was no significant difference between conditions, $t(17.2)=1.608, p=.126$. Average performance for the familiar items (i.e., those seen during the training phase) across blocks is displayed in Figure 2A. The average accuracy (collapsed across blocks) for both the rule $(M=.923, S D=.083, t(23)=25.111, p<.001)$ and similarity $(M=.763, S D=.129 ; t(13)=7.616, p<$ .001) groups was significantly above chance, although as in the training phase, rule-based participants had higher accuracy than similarity-based participants, $t(19.3)=$ $4.151, p<.001$. Median RTs were nonsignificantly longer in the similarity group (1208 msec) than the rule group (1060 msec), $t(18.4)=1.78, p=.09$.

Of particular importance, given our interest in generalization strategies, both the rule and similarity groups used their preferred strategy significantly above chance levels (rule group, $M=.862, S D=.106, t(24)=16.691$, $p<.001$; similarity group, $M=.825, S D=.095, t(13)=$ $12.868, p<.001$ ) for the critical test items (see Figure $2 \mathrm{~B}$ ), and there was no significant difference in strategyconsistent responding between groups, $t(29.3)=1.074$, $p=.292$, with substantial evidence for the null, BF (Bayes factor) $=0.25 .^{2}$ Median RTs were nonsignificantly shorter in the similarity group (1119 msec) than the rule group (1294 msec), $t(22.3)=1.86, p=.08$.

Although participants were classified on the basis of being either rule-consistent or similarity-consistent collapsed across all critical items, it does not necessarily follow that both the critical compound and element stimuli show this pattern. We therefore consider generalization to compound and element stimuli separately as in past work (e.g., Wills et al., 2011; Shanks \& Darby, 1998). The mean probability of predicting an allergic reaction to the critical compound stimuli (i.e., IJ and $\mathrm{MN}$ ) is shown in Figure 3A. As expected, there was a significant interaction between strategy used and stimulus type, $F(1$, $36)=382.02, p<.001$. No other main effects or interactions were significant ( $p s>.25$ ). The rule group showed rule-consistent generalization to compounds, $t(23)=$ $25.87, p<.001$, whereas the similarity group showed

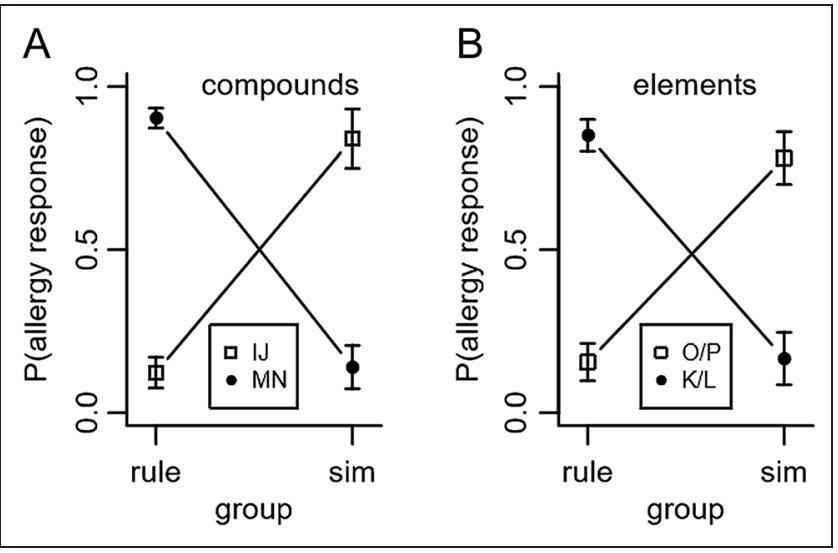

Figure 3. (A) Mean probability of predicting an allergic reaction for the critical compound stimuli. (B) Mean probability of predicting an allergic reaction for the critical element stimuli. Also shown are difference-adjusted 95\% confidence intervals for the between-subject effects (Baguley, 2012). 
similarity-consistent generalization, $t(13)=8.21, p<.001$. Median RTs were nonsignificantly shorter in the similarity group (1313 $\mathrm{msec}$ ) than the rule group (1457 msec), $F(1,36)=1.29, p=.26$. No other effects were significant $(p s>.12)$.

Figure $3 \mathrm{~B}$ shows the probability of predicting an allergic reaction to the critical element stimuli (i.e., $\mathrm{K} / \mathrm{L}$ and $\mathrm{O} / \mathrm{P})$. There was again a significant interaction between strategy used and stimulus type, $F(1,36)=201.80, p<$ .001 . No other main effects or interactions were significant $(p s>.4)$. The rule group showed rule-consistent generalization to elements, $t(23)=11.75, p<.001$, whereas the similarity group showed similarity-consistent generalization, $t(13)=9.43, p<.001$. Median RTs were nonsignificantly shorter in the similarity group (1123 msec) than the rule group (1289 msec), $F(1,36)=$ $3.96, p=.054$. No other main effects or interactions were significant $(p s>.06)$.

\section{Imaging Analyses}

\section{Training}

A contrast comparing all trials and groups against the implicit baseline revealed extensive activation including diverse regions of the bilateral $\mathrm{pFC}$, bilateral parietal lobes, and bilateral occipital lobes (see Figure 4A). We then compared performance on early training (Blocks 1-3) to late training (Blocks 3-6) for all trials and participants. A large cluster including the caudate head and body, which have been extensively linked to category learning (e.g., Seger, 2008), and the thalamus, was activated more early in training than later in training (peak voxel: $x=16, y=-32, z=16$ ). Conversely, the right inferior frontal gyrus (peak voxel: $x=24, y=25, z=$ -5 ) and the anterior cingulate/medial prefrontal gyrus (peak voxel: $x=8, y=30, z=22$ ) were activated more late in training than early in training.

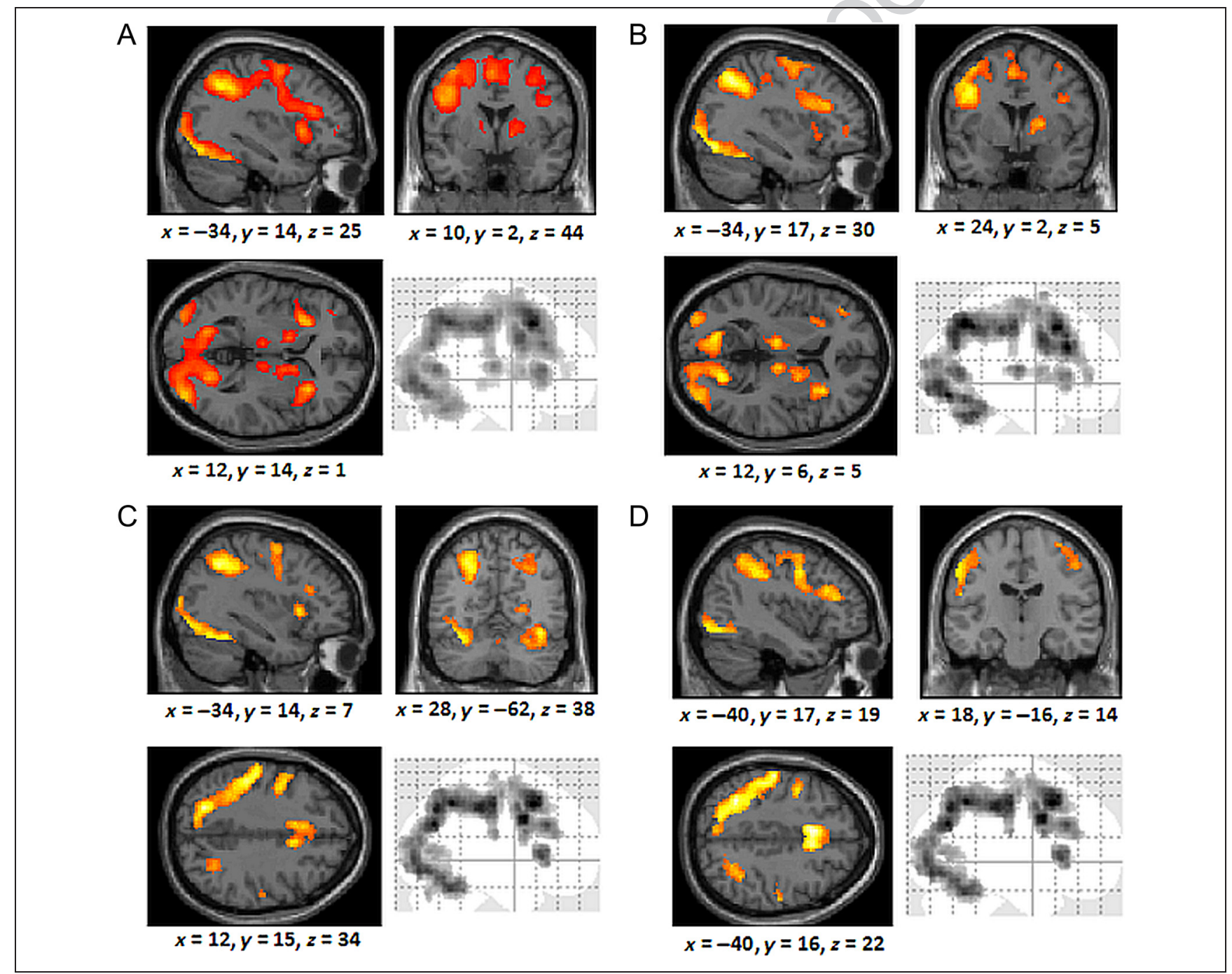

Figure 4. (A) Brain regions activated across all trials and all participants during training. (B) Regions activated by correct responses by the rule group during training. (C) Regions activated by correct responses in the similarity group during training. (D) Common brain regions activated by correct responses in the similarity and rule groups. The coordinates indicate the origin for the image displayed. Lighter colors indicate higher $z$ scores. 
Table 1. Regions Commonly Activated by Rule-based and Similarity-based Generalization in the Training Phase

\begin{tabular}{|c|c|c|c|c|c|c|}
\hline \multirow[b]{2}{*}{ Region } & \multirow[b]{2}{*}{ Cluster Size } & \multirow[b]{2}{*}{$B A$} & \multicolumn{3}{|c|}{ Talairach Coordinates } & \multirow[b]{2}{*}{$z$ Score } \\
\hline & & & $x$ & $y$ & $z$ & \\
\hline Left anterior cingulate & 1176 & 32 & -6 & 18 & 40 & 6.59 \\
\hline Right anterior cingulate & & 32 & 6 & 16 & 40 & 5.98 \\
\hline Left medial frontal gyrus & & 32 & -8 & 12 & 45 & 5.89 \\
\hline Left middle frontal gyrus & 5721 & 9 & -50 & 7 & 29 & 6.40 \\
\hline Left precuneus & & 19 & -28 & -67 & 29 & 6.36 \\
\hline Left inferior parietal lobe & & 40 & -32 & -50 & 43 & 6.34 \\
\hline Right inferior occipital gyrus & 3147 & 18 & 38 & -82 & -4 & 5.83 \\
\hline Right occipital lobe & & 19 & 28 & -74 & -5 & 5.71 \\
\hline Right middle occipital gyrus & & 18 & 36 & -84 & 2 & 5.66 \\
\hline Left insula & 185 & 13 & -30 & 20 & 1 & 5.65 \\
\hline Left inferior frontal gyrus & & 47 & -30 & 25 & -5 & 4.90 \\
\hline Right inferior frontal gyrus & 196 & 45 & 32 & 24 & 4 & 5.34 \\
\hline Right superior parietal lobe & 406 & 7 & 32 & -52 & 45 & 4.68 \\
\hline Right precuneus & & 7 & 18 & -62 & 49 & 4.19 \\
\hline Right superior parietal lobe & & 7 & 32 & -58 & 40 & 4.19 \\
\hline Right precentral gyrus & 354 & 4 & 48 & -11 & 48 & 4.66 \\
\hline Right precentral gyrus & & 6 & 34 & -14 & 62 & 4.18 \\
\hline Right precentral gyrus & & 6 & 40 & -7 & 52 & 3.87 \\
\hline
\end{tabular}

All activations significant at $p<.001$. Indented rows indicate voxels in the same cluster as the nonindented row above them. BA $=$ Brodmann's area.

For the rule group, comparing correct responses against the baseline revealed extensive activation including in the $\mathrm{pFC}$, parietal lobes, and the occipital lobes (Figure 4B). Similar regions were recruited by the similarity group (Figure 4C). This extensive common overlap of activation was confirmed by a conjunction analysis (Figure 4D;
Table 1). When directly comparing the groups, no regions were more activated by the similarity group than the rule group; however, the bilateral posterior cingulate/precuneus (cluster size: 166 voxels) was engaged more in the rule group than the similarity group (Figure 5A). An exploratory analysis of this rule-similarity contrast, with
Figure 5. (A) Brain regions more activated by the rule group than the similarity group across all blocks of training. (B) Brain regions more activated by rule-based responders than similarity responders across the second half of training. The coordinates indicate the origin for the image displayed. Lighter colors indicate higher $z$ scores.

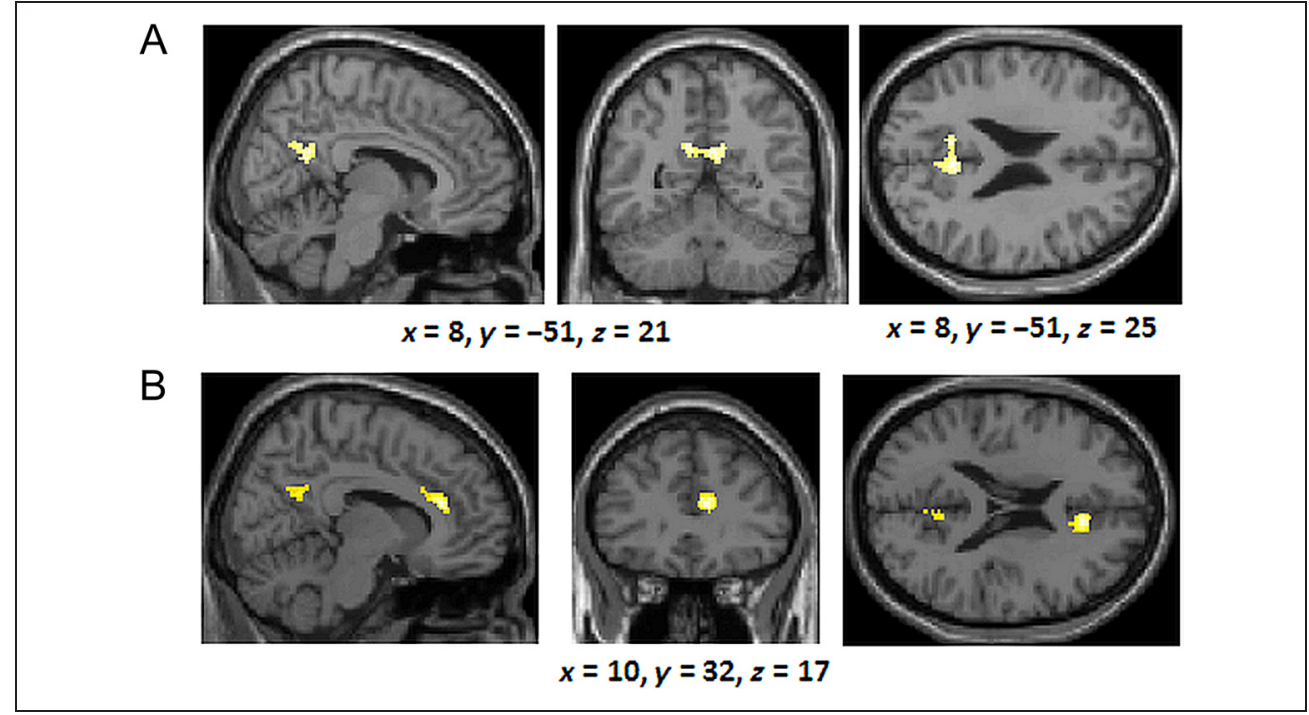


more liberal thresholds ( $p<.001,25$ contiguous voxels) and which should consequently be taken with caution, revealed two clusters in right middle frontal gyrus (first cluster, peak voxel $x=32, y=24, z=15$, cluster size: 41 voxels; second cluster, peak voxel, $x=28, y=23, z=$ 39, cluster size: 50 voxels).

No differences emerged between groups when considering just the first half of training. When looking at the second half of training alone, there were again no areas more activated by the similarity group than the rulebased group. However, the bilateral posterior cingulate/ precuneus was again activated more by the rule group than the similarity group, and the right anterior cingulate/medial frontal gyrus was also engaged (see Figure 5B).

\section{Test}

Critical trials (generalization). A number of brain regions were activated by similarity responders including bilateral inferior and superior parietal lobes, right middle occipital gyrus, and left medial frontal gyrus (Figure 6A). Rule-based responders engaged the left superior parietal lobes, bilateral inferior parietal lobes, bilateral middle frontal gyrus, left medial frontal gyrus, right inferior frontal gyrus, and bilateral occipital lobes (Figure 6B). A conjunction analysis (see Figure $6 \mathrm{C}$; Table 2 ) revealed extensive common overlap of activation between the similarityand rule-based participants, which included the left supe- rior parietal lobes, bilateral inferior parietal lobes, bilateral medial frontal gyrus, left middle frontal gyrus, and the bilateral occipital gyrus.

Next, we directly contrasted brain activation between the similarity and rule groups. In contrast to the extensive common activation, no differences were identified in whole-brain analyses. However, in the exploratory ROI analyses (comprising the pFC, right inferior parietal lobes, and bilateral occipital lobes, with thresholds of $p<.001$ and 64 contiguous voxels), we found that the right middle frontal gyrus (see Figure 7A; BA 9) was activated more for the rule group than the similarity group (in the same region as identified by the exploratory analysis documented in the rule-similarity comparison for the training phase). In contrast, we observed greater activation in the anterior medial frontal lobes (BA 10) and the right inferior parietal lobes (BA 40) for the similarity group compared with the rule-based group (Figure 7B).

Element versus compound critical stimuli. As a supplementary question, we assessed whether there were activation differences in the element (i.e., $\mathrm{K} / \mathrm{L}$ and $\mathrm{O} / \mathrm{P}$ ) and compound (i.e., IJ and $\mathrm{MN}$ ) critical trials. As before, only trials that were consistent with the preferred strategy of the participants (i.e., rule- or similarity-based) were included. For the rule group, there was greater activation in the occipital lobes/cerebellum and the left caudate body for the compound stimuli than for the

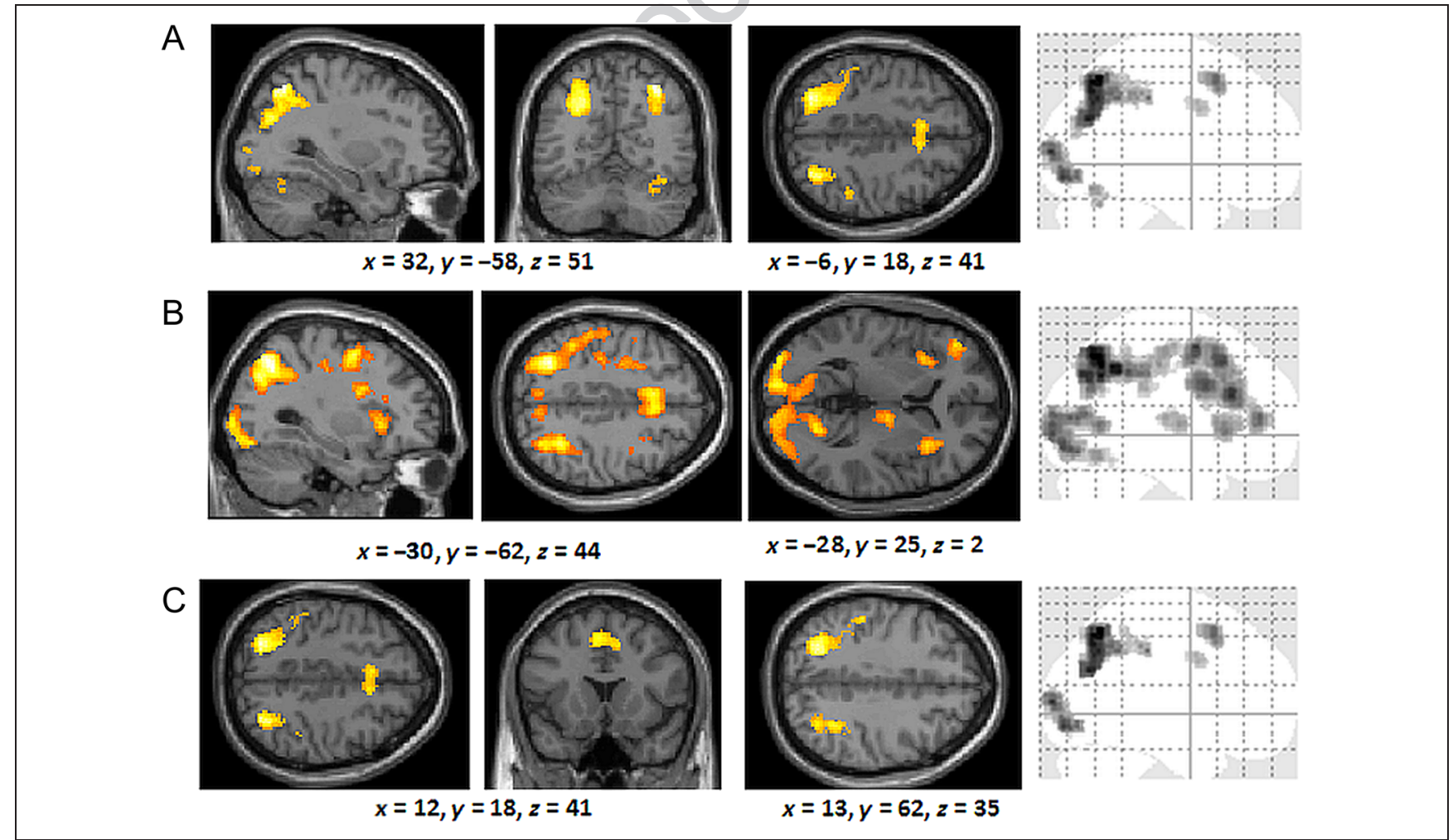

Figure 6. (A) Brain regions significantly activated by similarity responders during the critical trials. (B) Brain regions significantly activated by rule-based responders during the critical trials. (C) Common brain regions activated by similarity-and rule-based responders during the critical trials. The coordinates indicate the origin for the image displayed. Lighter colors indicate higher $z$ scores. 
Table 2. Regions Commonly Activated by Rule-based and Similarity-based Generalization in the Critical Generalization Trials

\begin{tabular}{|c|c|c|c|c|c|c|}
\hline \multirow[b]{2}{*}{ Region } & \multirow[b]{2}{*}{ Cluster Size } & \multirow[b]{2}{*}{$B A$} & \multicolumn{3}{|c|}{ Talairach Coordinates } & \multirow[b]{2}{*}{$z$ Score } \\
\hline & & & $x$ & $y$ & $z$ & \\
\hline Right superior parietal lobe & 566 & 7 & 32 & -58 & 51 & 5.40 \\
\hline Right precuneus & & 19 & 30 & -68 & 29 & 4.77 \\
\hline Right inferior parietal lobe & & 40 & 34 & -50 & 41 & 4.36 \\
\hline Left superior parietal lobe & 1040 & 7 & -26 & -62 & 44 & 5.09 \\
\hline Left precuneus & & 7 & -26 & -67 & 27 & 5.05 \\
\hline Left parietal lobe & & 39 & -28 & -62 & 36 & 4.97 \\
\hline Left middle occipital gyrus & 556 & 18 & -26 & -91 & 10 & 4.82 \\
\hline Left middle occipital gyrus & & 18 & -26 & -84 & -4 & 4.74 \\
\hline Left inferior occipital gyrus & & 19 & -38 & -76 & -5 & 4.23 \\
\hline Right middle occipital gyrus & 425 & 18 & 24 & -89 & 10 & 4.44 \\
\hline Right middle occipital gyrus & & 18 & 16 & -94 & 14 & 4.21 \\
\hline Right occipital lobe & & 17 & 22 & -8 & -2 & 3.97 \\
\hline Left medial frontal gyrus & 329 & 6 & -2 & 16 & 45 & 4.40 \\
\hline Right medial frontal gyrus & & 6 & & 16 & 42 & 3.94 \\
\hline Left middle frontal gyrus & 104 & 9 & -50 & 4 & 33 & 3.63 \\
\hline Left precentral gyrus & & 6 & -38 & 0 & 33 & 3.46 \\
\hline
\end{tabular}

All activations significant at $p<.001$. Indented rows indicate voxels in the same cluster as the nonindented row above them. BA $=$ Brodmann's area.

element stimuli (see Figure 8A). In contrast, no regions were more activated for the element stimuli than the compound stimuli. For the similarity group, no areas were more active for the compound stimuli than the element stimuli, although the occipital lobes were, as for the rule-based group, activated at lower thresholds (this could reflect, in part, the smaller sample size of the similarity group compared with the rule group). However, the left precentral/postcentral gyrus was activated more for the element stimuli than the compound stimuli (Figure 8B). When comparing the rule and similarity groups, no significant differences emerged.

Familiar items. Although not our primary focus, we also examined the brain activation for the familiar items to supplement the critical generalization trials analyses. Participants in the similarity-based group activated a diverse set of regions including bilateral occipital gyrus, left inferior parietal lobes, and bilateral middle frontal gyrus (Figure 9A). Rule-based generalizers recruited the
Figure 7. (A) Brain regions more activated by the rule-based responders than similarity responders for the critical trials. (B) Brain regions more activated by similarity responders than rule-based responders for the critical trials. The coordinates indicate the origin for the image displayed. Lighter colors indicate higher $z$ scores.

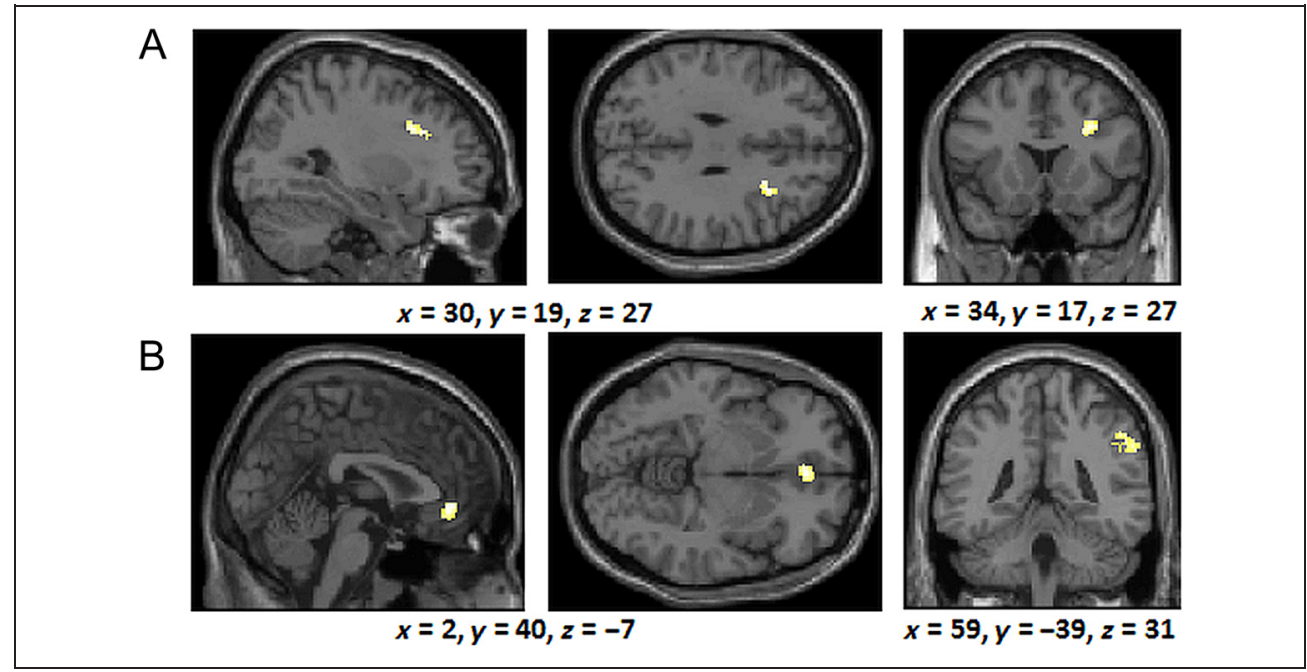


Figure 8. (A) Regions more activated by compound items than element items during the critical generalization trials for the rule-based group. (B) Regions more activated by element items than compound items during the critical generalization trials for the similarity group.

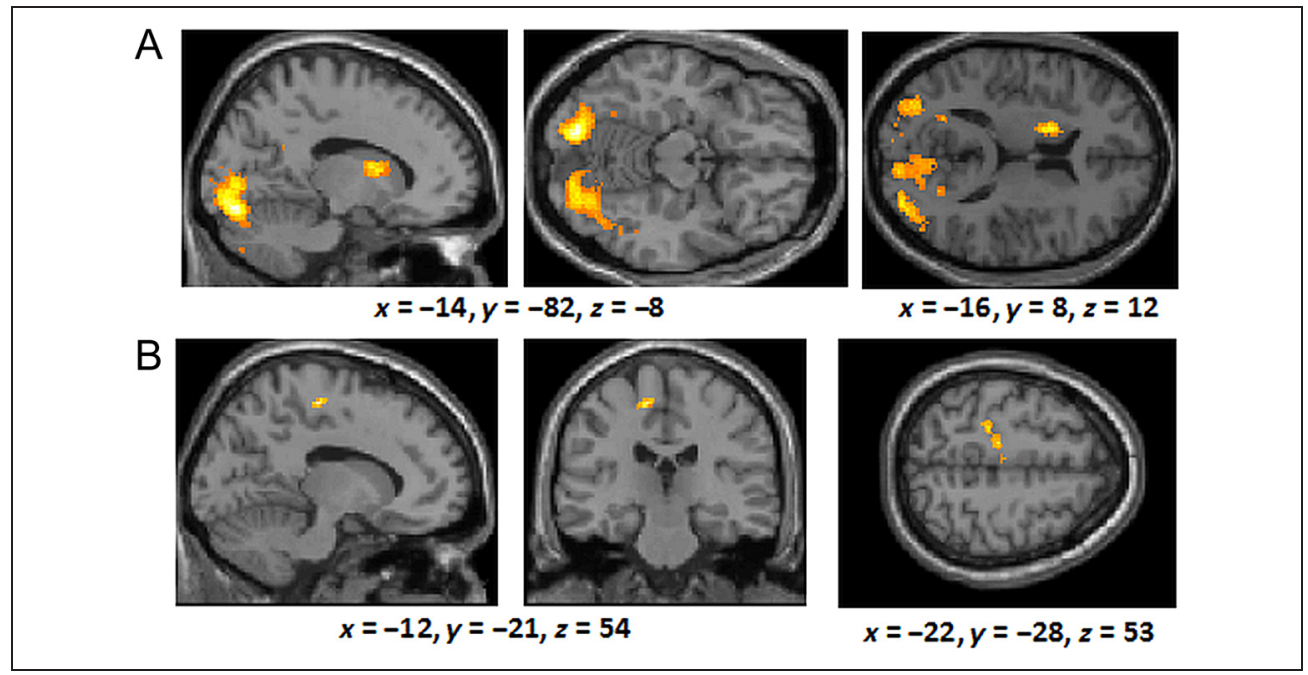

bilateral occipital lobes, the left superior parietal lobes, and the left inferior and middle frontal gyrus (Figure 9B). A conjunction analysis indicated common activation between the similarity- and rule-based responders in the bilateral occipital lobes, the left superior parietal lobes, and left postcentral gyrus (see Figure 9C; Table 3). This pattern is, broadly speaking, similar to what we observed in the training phase with the same stimuli.
No differences emerged between the rule and similarity groups in either whole-brain or our exploratory ROI analyses. This is perhaps not that surprising as these analyses are considerably less sensitive than the analogous critical trials analyses given that they do not directly measure generalization and one cannot determine at the individual trial level whether a response is rule or similarity consistent. As a further exploratory analysis, we used the

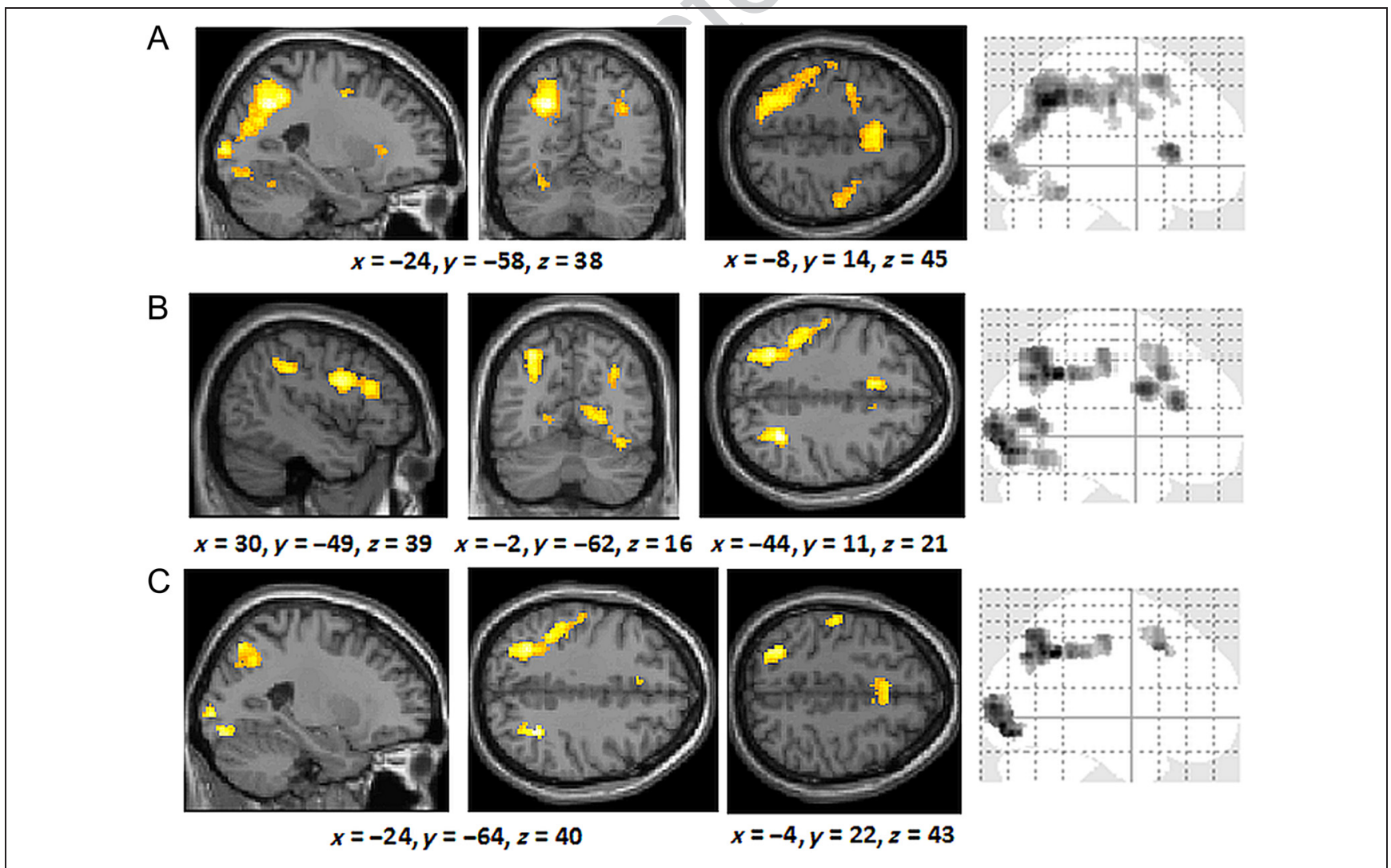

Figure 9. (A) Brain regions engaged by the similarity responders for the familiar items during test. (B) Brain regions engaged by the rule-based responders for the familiar items during test. (C) Brain regions commonly engaged by the similarity- and rule-based responders for the familiar items during test. The coordinates indicate the origin for the image displayed. Lighter colors indicate higher $z$ scores. 
Table 3. Regions Commonly Activated by the Rule and Similarity Groups during Test for the Familiar Items

\begin{tabular}{|c|c|c|c|c|c|c|}
\hline \multirow[b]{2}{*}{ Region } & \multirow[b]{2}{*}{ Cluster Size } & \multirow[b]{2}{*}{$B A$} & \multicolumn{3}{|c|}{ Talairach Coordinates } & \multirow[b]{2}{*}{ z Score } \\
\hline & & & $x$ & $y$ & $z$ & \\
\hline Right superior parietal lobe & 154 & 7 & 30 & -49 & 39 & 5.37 \\
\hline Right parietal lobe & & 39 & 30 & -56 & 38 & 4.70 \\
\hline Left middle occipital gyrus & 565 & 18 & -36 & -86 & -2 & 5.17 \\
\hline Left middle occipital gyrus & & 18 & -28 & -80 & -6 & 5.08 \\
\hline Left cuneus & & 17 & -14 & -91 & 8 & 5.01 \\
\hline Left superior parietal lobe & 858 & 7 & -30 & -56 & 40 & 5.07 \\
\hline Left precuneus & & 19 & -28 & -68 & 38 & 4.88 \\
\hline Left superior parietal lobe & & 7 & -30 & -62 & 49 & 4.81 \\
\hline Right middle occipital gyrus & 564 & 18 & 14 & -91 & 14 & 4.47 \\
\hline Right middle occipital gyrus & & 18 & 38 & -82 & 1 & 4.44 \\
\hline Right middle occipital gyrus & & 18 & 30 & -87 & 4 & 4.33 \\
\hline Left anterior cingulate & 244 & 32 & -8 & 17 & 38 & 4.45 \\
\hline Right superior frontal gyrus & & 8 & 2 & 18 & 47 & 3.86 \\
\hline Left superior frontal gyrus & & 6 & -6 & 8 & 49 & 3.54 \\
\hline
\end{tabular}

All activations significant at $p<.001$. Indented rows indicate voxels in the same cluster as the nonindented row above them. BA $=$ Brodmann's area.

WFU Pickatlas (Maldjian et al., 2003) to construct a mask containing all regions identified in the similarity-rule analysis of the critical items previously reported (Figure 7B) and identified whether there was any activation in these areas for the familiar items at thresholds of $p<$ .005 (uncorrected) and 10 contiguous voxels. This analysis revealed activation in the right inferior parietal lobes (peak voxel $x=59, y=-37, z=31$, cluster size: 37 voxels). We also conducted the same type of analysis for the right middle frontal gyrus region (Figure $7 \mathrm{~A}$ ) that was more activated in the rule than the similarity condition for the critical generalization trials but did not detect any activation here.

Participants with a mixture of rule- and similarityconsistent responses. Fourteen participants met the learning criterion for training but showed no clear pattern of similarity- or rule-based responding for the critical generalization trials and were consequently excluded from the analyses above. Nevertheless, given that these participants had a mixture of both types of strategy (ruleconsistent, $M=.501$; similarity-consistent, $M=.499$ ), they
Figure 10. (A) Regions activated by similarity-consistent responses for the group who displayed a mixture of both strategies. (B) Regions activated by rule-consistent responses for the group who displayed a mixture of both strategies.

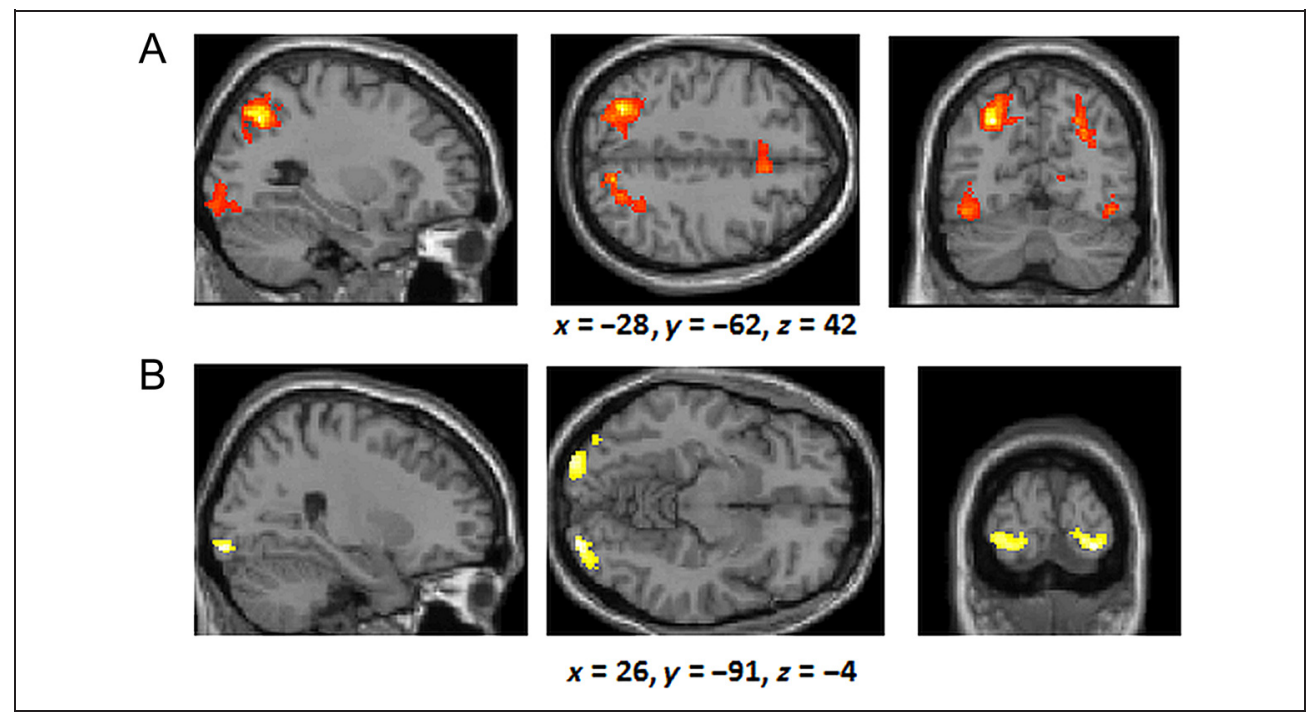


provide an opportunity to look at the neural correlates of rule- and similarity-based responding within participants.

Similarity-consistent responses evoked activation in the bilateral anterior cingulate/medial frontal gyrus, the left superior parietal lobe, and the bilateral occipital lobes (Figure 10A). Rule-based responding also activated bilateral occipital lobes (see Figure 10B). We did not, however, detect any significant activation elsewhere although exploratory analyses with more liberal thresholds $(p<$ .001 and 25 contiguous voxels) revealed a similar pattern of activation to what we observed with the similarity responders. We suspect the reduced activation here compared with the corresponding analysis for the consistent rule-based sorters reflects the lower number of participants and trials in the current analysis. We found no indication of any differences between strategies in either whole-brain or exploratory ROI analyses.

\section{DISCUSSION}

This study used a negative and positive patterning design originally developed by Shanks and Darby (1998; see also Wills et al., 2011) to compare the brain activation of rule and similarity generalization. Participants were divided into either rule-based or similarity-based generalizers according to their responses during the critical items at test. Importantly, participants in both groups were highly consistent and did not differ in their ability to follow their preferred strategy. There was extensive overlap of activation between the rule- and similarity-based groups, but at the same time there were regions that were differentially activated by the two strategies. We discuss the most notable aspects of our findings below.

In the training phase, there was extensive overlap between groups including diverse regions of the $\mathrm{pFC}$, the parietal lobes, and the occipital lobes. Differences between strategies, in contrast, were more restricted-no regions were more active in the similarity group than the rule group, although the posterior cingulate/precuneus and the anterior cingulate/medial prefontal cortex (in the second half of training) were more active in the rule group than the similarity group. The greater posterior cingulate/ precuneus activation is perhaps somewhat surprising, although these regions have been implicated in rulebased category learning (Milton \& Pothos, 2011). The anterior cingulate activation is in line with the key role this region is thought to play in rule selection in COVIS's rulebased system (Ashby et al., 1998).

In the test phase, there was again considerable common overlap in the regions activated by the rule and similarity groups for both the critical generalization trials and the familiar trials. As before, areas activated included regions of the pFC (including the middle frontal gyrus), bilateral parietal lobes, and bilateral occipital lobes. These regions have all previously been implicated in categorization tasks (e.g., Carpenter et al., in press; Milton et al., 2009). For example, the bilateral parietal lobes have been heavily implicated in both explicit and implicit classification of dot patterns (Aizenstein et al., 2000) and stimulus generalization (Seger, Braunlich, Wehe, \& Liu, 2015). Furthermore, our results add to the growing body of evidence that diverse regions of the $\mathrm{pFC}$ are involved in categorization (e.g., Milton \& Pothos, 2011; Koenig et al., 2005; Grossman et al., 2002; Seger \& Cincotta, 2002). The common activation shared by the rule and similarity groups across both the category learning and generalization components of this task is consistent with the idea that both strategies share a number of common, interrelated processes, such as stimulus processing, response selection, stimulus-response mappings, feedback processing (whether it is an external signal as in training or more internally generated as is likely in test), uncertainty, and attentional and working memory demands to name just a few likely candidates.

Although no differences were identified between generalization strategies in whole-brain analyses, exploratory ROI analyses provided evidence for dissociable activation between the rule and similarity groups. As predicted, the rule-based generalizers activated the right middle frontal cortex to a greater extent than the similarity-based generalizers. This was in keeping with the trend from the training phase for there to be greater activation in the right $\mathrm{pFC}$ in the rule group than the similarity group. In contrast, the similarity-based generalizers preferentially recruited the anterior medial prefrontal lobe and the right inferior parietal lobes.

The greater right middle frontal gyrus activation in rule-based generalizers than similarity-based generalizers is consistent with a broad range of previous work indicating that the middle frontal lobes is a critical site for rulebased categorization (e.g., Milton \& Pothos, 2011; Seger \& Cincotta, 2002, 2005; Tracy et al., 2003; Grossman et al., 2002; Patalano et al., 2001) and, more generally, its role is well established in working memory processing (Owen, 2000). This pattern of findings is, therefore, in line with the idea that the "opposites" rule is the result of deliberative, rule-based processes, rather than being driven by a non-rule-based, associatively mediated system (Maes et al., 2015).

Turning to the regions preferentially linked to similarity responding, the right inferior parietal lobes were identified by Grossman et al. (2002) as being linked to similarity judgments, and they suggested that it had a role in overall feature configuration processing. Alternatively, it could reflect recollection-based memory processes, which are often observed in this region (e.g., Milton, Muhlert, Butler, Benattayallah, \& Zeman, 2011; Wheeler \& Buckner, 2004). This would be consistent with the idea that similarity processing places particular demands on the retrieval of past, related instances. We found that the anterior medial prefrontal lobe was activated more for the similarity than the rule group, in a strikingly similar location to that observed by Koenig et al. (2005) in their analogous comparison. Our explanation for this result 
is similar to Koenig et al.'s-the activation in this region may reflect the greater dependence on retrieving specific exemplars from long-term memory in the similarity-based condition rather than having generalization supported by an abstract rule.

Of course, there is always a danger in linking specific brain regions to a particular function and in knowing whether the regions identified are essential for the strategy used. For example, the fact that both the similarity and rule groups activated different parts of the pFC more than the other strategy appears to challenge any clear-cut narrative that rule generalization requires higher-order, deliberative processes whereas similarity generalization requires more automatic, nondeliberative processes. One intriguing future approach may be to further explore the regions where differences emerged (i.e., the right middle frontal gyrus for the rule generalizers and the anterior medial frontal lobe and right inferior parietal lobes for the similarity generalizers) using TMS. For example, according to our results, one might expect that stimulating the right middle frontal gyrus would disrupt rule generalization but leave similarity generalization intact, whereas stimulating the anterior prefrontal lobe (or right inferior parietal lobes) might impair similarity generalization but not rule generalization. Furthermore, if the greater activation in the anterior medial $\mathrm{pFC}$ reflects a greater reliance on retrieving past instances in similarity generalization than rule generalization, then one might expect that stimulating this region would disrupt performance in a task using similar stimuli which more directly assesses memory capacity.

Regardless of the precise role that these regions may play, using the Shanks-Darby procedure to examine the neural correlates of rule and similarity generalization makes a valuable new contribution to the area because it overcomes some problems that mar the paradigms used in previous related imaging studies. In particular, many studies in this area confound rule-based and similarity-based learning with single versus multidimensional learning (e.g., Milton \& Pothos, 2011; Nomura et al., 2007; Tracy et al., 2003). Furthermore, all previous studies investigating this issue index rule-based learning through behavior that can also be produced by a simple associative mechanism that incorporates some process of selective attention (e.g., ALCOVE; Kruschke, 1992). In contrast, for the Shanks-Darby procedure, there is no simple associative model that can explain both the similarity- and rule-based generalization observed and the same number of dimensions is relevant in both rule- and similarity-based learning.

There are other notable differences between the Shanks-Darby procedure and the other tasks previously used (e.g., Nomura et al., 2007; Koenig et al., 2005; Tracy et al., 2003; Grossman et al., 2002), which may have impacted the results obtained. For instance, the ShanksDarby task enables one to partition items into generalization trials (items not encountered during training) and familiar trials (which had previously been learned during training). In contrast, other studies have either used cat- egory learning (e.g., Carpenter et al., in press; Nomura et al., 2007) or category decision-making (e.g., Milton et al., 2009; Grossman et al., 2002) procedures where there is no generalization phase or used imaging analyses that combine old training items with generalization trials (e.g., Koenig et al., 2005). Given that, in the test phase, we only observed evidence for differences in the generalization trials and not in the familiar trials, this could be an important distinction.

A second important difference is that previous studies typically use multidimensional stimuli that possess either binary (e.g., Milton et al., 2009; Tracy et al., 2003) or continuous values (e.g., Carpenter et al., in press; Nomura et al., 2007) on a particular dimension. In contrast, our stimuli have discrete components (e.g., A, B, AB, etc.) that are either present or absent. This distinction has not been systematically investigated but could potentially have an important impact on the pattern of results obtained. Clearly, in the future, it would be of value to further explore rule and similarity learning under a more diverse range of conditions to build up a broader understanding of how the two types of strategies relate to each other.

Although the Shanks-Darby procedure appears to have a number of strengths, one potential limitation is that, although the rule and similarity groups were well matched in their consistency of applying their preferred strategy for the critical generalization trials, the rule group significantly outperformed the similarity group for the familiar training trials (this is the same pattern that Shanks \& Darby, 1998, found). It is worth noting, though, that the familiar trials were analyzed separately from the critical generalization trials where the neural differences emerged, which should attenuate any influence this difference would have on our results. Furthermore, differences between groups for the familiar items (where only correct responses were considered) were extremely restricted, which suggests that performance difference between groups had little impact on the pattern of activation. Nevertheless, in future work with the Shanks-Darby procedure, it would be desirable to have the groups better matched on performance for the familiar trials. One way of doing this could be to introduce a learning criterion during the training phase, which has been shown to better equate groups on the familiar test items in this procedure (Wills et al., 2011).

Another notable aspect of our results is that the key comparison between rule and similarity generalization was between participants. While we looked at this from a within-subject perspective as well by considering those participants who displayed a mixture of strategies, these analyses were not particularly revealing. We suspect that this was due to these participants randomly responding on the critical trials and/or that there were insufficient trials of each type to reliably detect any differences. In the future, one could potentially further increase the number of critical generalization trials in the procedure and/or use a combination of more effective instructions 
than we used with training outside the scanner to ensure that participants produce a good mixture of rule and similarity generalization responses.

Another limitation of this study is that, although the differences between generalization strategies were consistent with our a priori predictions and in line with past related work, it must be acknowledged that these differences were not identifiable in whole-brain analyses and could only be detected in more exploratory, post hoc analyses. Clearly, it would be valuable for future work to try and replicate our basic pattern of results.

Nevertheless, our finding of differences in activation between rule and similarity generalization (albeit only in exploratory analyses) is in line with recent behavioral and comparative studies that have been conducted with the Shanks-Darby procedure. Specifically, Wills et al. (2011) found that participants who completed the training session under a concurrent load went on to produce significantly less rule-based generalization than participants who undertook training under no load. Wills et al. suggested that this is consistent with the idea that discovering the "opposites" rule requires considerable working memory capacity and if this is not available that participants will fail to transition from using a similarity approach to a rule-based approach. Related to this, Maes et al. (2015) found that, although humans (under no concurrent load) could readily discover the opposites rule, both pigeons and rats were unable to do so and relied on similarity generalization. This is consistent with the idea that pigeons and rats may be forced to rely on the similarity system whereas humans also have access to a rule-based system. Our findings complement these two recent behavioral studies by identifying specific neural correlates that are associated with rule and similarity-based generalization.

However, although our results make a novel and valuable contribution to the area, our findings stop some way short of providing clear evidence for qualitatively separable generalization systems by any reasonable definition. For instance, neural differences in themselves should not be taken as evidence for separable systems given that past work has shown that items within the same category can provoke differential activation (e.g., Davis \& Poldrack, 2014; DeGutis \& D'Esposito, 2009; Grinband, Hirsch, \& Ferrera, 2006). What may be more compelling evidence for separable learning systems would be large differences in activation in regions that are not also activated by the other strategy. This does not appear to be the case in this study, where the differences in generalization were restricted and located close to regions that were activated in training and test by both strategies. Furthermore, the commonalities in activation between strategies clearly outweigh the differences.

One potentially fruitful way of viewing the current data, then, is to consider category learning and stimulus generalization as cognitively complex processes comprising a number of subcomponents (e.g., stimulus processing, hypothesis testing, decision-making, feedback process- ing, etc.) many of which are likely to be shared by rule and similarity strategies. Furthermore, one strategy may place more of an emphasis on one of these subprocesses than the other strategy does. For example, the "opposites" rule needed for rule generalization appears likely to place particular demands on working memory capacity and rule formation that do not appear to be needed to the same extent for similarity generalization and this may require increased activation of the right middle frontal gyrus. Conversely, a similarity strategy may, for example, impose higher memory demands than the rule condition (where there may be more of an emphasis on abstract rules), which could lead to greater engagement of the anterior medial prefrontal lobes. Of course, our hypotheses as to what particular role these brain regions play may not be correct but as others have recommended (e.g., Davis, Love, \& Preston, 2012) trying to link brain regions to specific functions of the learning process may be at least as profitable an approach as focusing on the more general and contentious question of whether data is more in line with single-or multiple-system accounts. We suggest that further examination of the Shanks-Darby procedure, with its notable strengths, could play an important role in further illuminating both these important questions.

\section{UNCITED REFERENCE}

Sloman, 1996

\section{Acknowledgments}

We thank the Experimental Psychology Society for their support and two anonymous reviewers for their insightful and constructive comments.

Reprint requests should be sent to Fraser Milton, Discipline of Psychology, University of Exeter, Exeter, EX4 4QG, United Kingdom, or via e-mail: f.n.milton@ex.ac.uk.

\section{Notes}

1. The raw imaging and behavioral data are available for interested readers at www.willslab.co.uk/exe10/index.html.

2. By convention, a BF of over three is interpreted as providing substantial evidence for the experimental hypothesis (Jeffreys, 1961), whereas a BF below a third provides substantial evidence for the null (Dienes, 2011). BF analysis requires an estimate of the mean expected difference under the experimental hypothesis; we estimated this from the observed difference for the familiar test items. Following Dienes (2011), the expected difference was modeled as a two-tailed normal distribution with a standard deviation equal to half the mean. Calculations were run using a custom script (Baguley \& Kaye, 2010) within R (R Core Team, 2015).

\section{REFERENCES}

Aizenstein, H. S., MacDonald, A. W., Stenger, V. A., Nebes, R. D., Larson, J. K., Ursu, S., et al. (2000). Complementary category learning systems using event-related functional MRI. Journal of Cognitive Neuroscience, 12, 977-987. 
Allen, S. W., \& Brooks, L. R. (1991). Specializing the operation of an explicit rule. Journal of Experimental Psychology: General, 120, 3-19.

Ashby, F. G., Alfonse-Reese, L. A., Turken, A. U., \& Waldron, E. M. (1998). A neuropsychological theory of multiple systems in category learning. Psychological Review, 105, 442-481.

Ashby, F. G., \& Maddox, W. T. (2011). Human category learning 2.0. Annals of the New York Academy of Sciences, 1224, 147-161.

Baguley, T., \& Kaye, D. (2010). Book review: Understanding psychology as a science: An introduction to scientific and statistical inference. British Journal of Mathematical and Statistical Psychology, 63, 695-698.

Brooks, L. (1978). Nonanalytic concept formation and memory for instances. In E. Rosch \& B. B. Lloyd (Eds.), Cognition and categorization. Hillsdale, NJ: Erlbaum.

Carpenter, K. L., Wills, A. J., Benattayallah, A., \& Milton, F. (in press). A comparison of the neural correlates that underlie rule-based and information-integration category learning. Human Brain Mapping. doi:10.1002/hbm.23259.

Davis, T., Love, B. C., \& Preston, A. R. (2012). Learning the exception to the rule: Model-based fMRI reveals specialized representations for surprising category members. Cerebral Cortex, 22, 260-273

Davis, T., \& Poldrack, R. A. (2014). Quantifying the internal structure of categories using a neural typicality measure. Cerebral Cortex, 24, 1720-1757.

DeGutis, J., \& D'Esposito, M. (2009). Network changes in the transition from initial learning to well-practiced visual categorization. Frontiers in Human Neuroscience, 3, 1-13.

Dienes, Z. (2011). Bayesian versus orthodox statistics: Which side are you on? Perspectives in Psychological Science, 6, 274-290.

Edmunds, C. E. R., Milton, F., \& Wills, A. J. (2015). Feedback can be superior to observational training for both rule-based and information-integration category learning. Quarterly Journal of Experimental Psychology, 68, 1203-1222.

Grinband, J., Hirsch, J., \& Ferrera, V. P. (2006). A neural representation of categorization uncertainty in the human brain. Neuron, 49, 757-763.

Grinband, J., Wager, T. D., Lindquist, M., Ferrera, V. P., \& Hirsch, J. (2008). Detection of time-varying signals in event-related fMRI designs. Neuroimage, 43, 509-520.

Grossman, M., Smith, E. E., Koenig, P., Glosser, G., DeVita, C., Moore, P., et al. (2002). The neural basis for categorization in semantic memory. Neuroimage, 17, 1549-1561.

Jeffreys, H. (1961). The theory of probability (3rd ed.). Oxford, UK: Oxford University Press.

Kemler Nelson, D. G. (1984). The effect of intention on what concepts are acquired. Journal of Verbal Learning \& Verbal Behavior, 23, 734-759.

Kiebel, S. J., Poline, J. B., Friston, K. J., Homes, A. P., \& Worsley, K. J. (1999). Robust smoothness estimation in statistical parametric maps using standardized residuals from the general linear model. Neuroimage, 10, 756-766.

Koenig, P., Smith, E. E., Glosser, G., DeVita, C., Moore, P., McMillan, C., et al. (2005). The neural basis for novel semantic categorization. Neuroimage, 24, 369-383.

Kruschke, J. K. (1992). ALCOVE: An exemplar-based connectionist model of category learning. Psychological Review, 99, 22-44.

Maes, E., De Filippo, G., Inkster, A. B., Lea, S. E. G., De Houwer, J., D'Hooge, R., et al. (2015). Feature- versus rule-based generalization in rats, pigeons and humans. Animal Cognition, 18, 1267-1284.

Maldjian, J. A., Laurienti, P. J., Burdette, J. B., \& Kraft, R. A. (2003). An automated method for neuroanatomic and cytoarchitectonic atlas-based interrogation of fMRI data sets. Neuroimage, 19, 1233-1239.

Milton, F., Longmore, C. A., \& Wills, A. J. (2008). Processes of overall similarity sorting in free classification. Journal of Experimental Psychology: Human Perception and Performance, 34, 676-692.

Milton, F., Muhlert, N., Butler, C. R., Benattayallah, A., \& Zeman, A. Z. J. (2011). The neural correlates of everyday recognition memory. Brain and Cognition, 76, 369-381.

Milton, F., \& Pothos, E. M. (2011). Category structure and the two learning systems of COVIS. European Journal of Neuroscience, 34, 1326-1336.

Milton, F., \& Wills, A. (2004). The influence of stimulus properties on category construction. Journal of Experimental Psychology: Learning, Memory \& Cognition, 30, 407-415.

Milton, F., Wills, A. J., \& Hodgson, T. L. (2009). The neural basis of overall similarity and single-dimension sorting. Neuroimage, 46, 319-326.

Newell, B. R., Dunn, J. C., \& Kalish, M. (2011). 6 Systems of category learning: Fact or fantasy? In $\mathrm{B}$. H. Ross (Ed.), The psychology of learning \& motivation (Vol. 54, pp. 167-215).

Newell, B. R., Moore, C. P., Wills, A. J., \& Milton, F. (2013). Reinstating the frontal lobes? Having more time to think improves implicit perceptual categorization: A comment on Filoteo, Lauritzen, and Maddox (2010). Psychological Science, 24, 386-389.

Nichols, T., Brett, M., Andersson, J., Wager, T., \& Poline, J. B. (2005). Valid conjunction inference with the minimum statistic. Neuroimage, 25, 653-660.

Nomura, E. M., Maddox, W. T., Filoteo, J. V., Ing, A. D., Gitelman, D. R., Parrish, T. B., et al. (2007). Neural correlates of rule-based and information-integration visual category learning. Cerebral Cortex, 17, 37-43.

Nomura, E. M., \& Reber, P. J. (2008). A review of medial temporal lobe and caudate contributions to visual category learning. Neuroscience and Biobehavioral Reviews, 32, 279-291.

Nosofsky, R. M., \& Johansen, M. K. (2000). Exemplar-based accounts of "multiple-system" phenomena in perceptual categorization. Psychonomic Bulletin \& Review, 7, 375-402.

Nosofsky, R. M., \& Kruschke, J. K. (2002). Single-system models and interference in category learning: Commentary on Waldron and Ashby (2001). Psychonomic Bulletin \& Review, 9. $169-174$

Owen, A. M. (2000). The role of the lateral frontal cortex in mnemonic processing: The contribution of functional imaging. Experimental Brain Research, 133, 33-43.

Patalano, A. L., Smith, E. E., Jonides, J., \& Koeppe, R. A. (2001). PET evidence for multiple strategies of categorization. Cognitive, Affective \& Behavioral Neuroscience, 1, 360-370.

R Core Team. (2015). R: A language and environment for statistical computing. Vienna: R Foundation for Statistical Computing. www.R-project.org/.

Rips, L. J. (1989). Similarity, typicality, and categorization. In S. Vosniadou \& A. Ortony (Eds.), Similarity and analogical reasoning (pp. 21-59). Cambridge, UK: Cambridge University Press.

Rosch, E., \& Mervis, C. B. (1975). Family resemblances: Studies in the internal structure of categories. Cognitive Psychology, 7, 573-605.

Seger, C. A. (2008). How do the basal ganglia contribute to categorization? Their roles in generalization, response selection, and learning via feedback. Neuroscience and Biobehavioral Reviews, 32, 265-278.

Seger, C. A., Braunlich, K., Wehe, H. S., \& Liu, Z. (2015). Generalization in category learning: The roles of representational and decisional uncertainty. Journal of Neuroscience, 35, 8802-8812. 
Seger, C. A., \& Cincotta, C. M. (2002). Striatal activity in concept learning. Cognitive Affective $\&$ Behavioral Neuroscience, 2, 149-161.

Seger, C. A., \& Cincotta, C. M. (2005). The roles of the caudate nucleus in human classification learning. Journal of Neuroscience, 25, 2941-2951.

Shanks, D. R., \& Darby, R. J. (1998). Feature- and rule-based generalization in human associative learning. Journal of Experimental Psychology: Animal Behavior Processes, 24, 405-415.

Sloman, S. A. (1996). The empirical case for two systems of reasoning. Psychological Bulletin, 119, 3-22.

Smith, E. E., Patalano, A. L., \& Jonides, J. (1998). Alternative strategies of categorization. Cognition, 65, 167-196.

Stanton, R. D., \& Nosofsky, R. M. (2013). Category number impacts rule-based and information-integration category learning: A reassessment of evidence for dissociable category-learning systems. Journal of Experimental Psychology: Learning, Memory, \& Cognition, 39, 1174-1191.

Talairach, J., \& Tournoux, P. (1988). Co-planar stereotaxic atlas of the buman brain. 3-Dimensional proportional system: An approach to cerebral imaging. Stuttgart, Germany: Thieme.

Tracy, J. I., Mohamed, F., Faro, S., Pinus, A., Tiver, R., Harvan, J., et al. (2003). Differential brain responses when applying criterion attribute versus family resemblance rule learning. Brain and Cognition, 51, 276-286.

Ward, T. B., \& Scott, J. (1987). Analytic and holistic modes of learning family-resemblance concepts. Memory \& Cognition, 15, 42-54.

Wheeler, M. E., \& Buckner, R. L. (2004). Functional-anatomic correlates of remembering and knowing. Neuroimage, 21, 1337-1349.

Wilkinson, D. T., Halligan, P. W., Henson, R. N. A., \& Dolan, R. J. (2002). The effects of interdistracter similarity on search processes in the superior parietal cortex. Neuroimage, 15, 611-619.

Wills, A. J., Graham, S., Koh, Z., Mclaren, I. P. L., \& Rolland, M. D. (2011). Effects of concurrent feature- and rule-based generalization in human contingency learning. Journal of Experimental Psychology: Animal Behavior Processes, 37, 308-316.

Wills, A. J., Inkster, A. B., \& Milton, F. (2015). Combination or differentiation? Two theories of processing order in classification. Cognitive Psychology, 80, 1-33.

Wills, A. J., Milton, F., Longmore, C. A., Hester, S., \& Robinson, J. (2013). Is overall similarity classification less effortful than single-dimension classification? Quarterly Journal of Experimental Psychology, 66, 299-318. 